\title{
A new empirical complex electrical resistivity model
}

\author{
M. Kavian ${ }^{1}$, E. C. Slob ${ }^{1}$, and W. A. Mulder ${ }^{2}$
}

\begin{abstract}
Macroscopic measurements of electrical resistivity require frequency-dependent effective models that honor the microscopic effects observable in macroscopic measurements. Effective models based on microscopic physics exist alongside with empirical models. We adopted an empirical model approach to modify an existing physical model. This provided a description of electrical resistivity as a function of not only frequency, but also water saturation. We performed two-electrode laboratory measurements of the complex resistivity on a number of fine and medium-grained unconsolidated sand packs saturated with water of three different salinities. For frequencies between 0.1 and $1 \mathrm{MHz}$, the data were fitted with the new model and compared to fits with Archie's law. Our model described the relaxation times and DC resistivity values as negative exponential functions with increasing water saturation. All data could be accurately described as a function of frequency and water saturation with nine parameters.
\end{abstract}

\section{INTRODUCTION}

Archie's law (Archie, 1942) relates the in situ bulk electrical resistivity of a sedimentary rock to its porosity and water saturation. For the real part $\rho_{r}$ of the complex resistivity $\rho=\rho_{r}-\mathrm{i} \rho_{i}$, it reads

$$
\rho_{r}=a \rho_{w} \phi^{-m} S_{w}^{-n}
$$

where $\rho_{r}$ represents the real part of the resistivity of the watersaturated sample, $\rho_{w}$ the resistivity of the pore fluid water, $\phi$ the porosity, $S_{w}$ the water saturation, and $a$ is the tortuosity factor. The symbol $\mathrm{i}$ denotes the imaginary unit. The subscripts $r$ and $i$ represent the real and the imaginary parts, respectively. The powers $m$ and $n$ are the cementation and saturation exponents, respectively. These powers can be found by fitting the real part of the resistivity data. The presence of a substantial fine-grained fraction in a sample in combination with a high water saturation leads to conduction on the grain surfaces (Waxman and Smits, 1968; Rink and Schopper, 1974; Park and Dickey, 1989). This means that the pore geometry has an influence on the bulk electrical resistivity, an effect that Archie's law does not capture. Frequency-domain electrical measurements on prepared samples provide an accurate estimate of the sample bulk resistivity.

Existing models that predict complex electrical resistivity either describe frequency dependence, such as the Cole-Cole model (Cole and Cole, 1941), or describe water saturation dependence, e.g. (Archie, 1942). If we could model frequency dependence and water saturation, it would be beneficial for oil field applications as well as environmental and engineering near-surface applications and in vadose zone hydrology. Several authors have studied this subject in the low-frequency range, below $1 \mathrm{kHz}$ (Ulrich and Slater, 2004; Titov et al., 2004; Binley et al., 2005; Cassiani et al., 2009; Ghorbani et al., 2009; Jougnot et al., 2010, among others). They found that the length scales of the polarization process change with fluid saturation. Binley et al. (2005) found that for a sandstone, the relaxation time of a Cole-Cole model decreases with decreasing saturation, but they did not give a functional relationship. Ghorbani et al. (2009) observed contradictory relationships, but also did not give a functional relationship. They used the double Cole-Cole model (Cole and Cole, 1941) to investigate the effect of desaturation on the relaxation time of clayrocks. They found that one of the two relaxation times was nearly constant during desaturation while the other increased for decreasing water saturation. They related textural changes to polarization processes associated with the bound water in the micropores.

Recently, Kavian et al. (2011) considered higher frequencies, above $100 \mathrm{kHz}$, and selected a simplified five-parameter double Cole-Cole model to fit the electrical permittivity data of unsaturated and unconsolidated sand packs. They used the model to describe the measured data as a function of frequency, with different parameters

Manuscript received by the Editor 20 August 2011; revised manuscript received 22 December 2011; published online 11 April 2012.

${ }^{1}$ Delft University of Technology, Department of Geotechnology, Section of Applied Geophysics and Petrophysics, Delft, The Netherlands. E-mail: m.kavian@tudelft.nl; e.c.slob@tudelft.nl.

${ }^{2}$ Delft University of Technology, Department of Geoscience and Engineering, Section of Applied Geophysics and Petrophysics, Delft, The Netherlands; Shell Global Solutions International BV, Rijswijk, The Netherlands.

(C) 2012 Society of Exploration Geophysicists. All rights reserved. 
for each saturation level and each salt concentration. Here, we present an electrical resistivity relaxation model that includes the saturation dependence. The imaginary part of the electrical resistivity of this model may have an additional dependence on pore size (Vinegar and Waxman, 1984), but porosity is not an explicit parameter in our model. The model should be useful for the interpretation of borehole logging data, as they have the same frequency range and physical scale as our experiments. Our model could have validity below the frequencies used here, but this is open to further research.

Below, we start with a description of the experiments, including the choice of samples and the analysis of the measured electrical resistivity data. The samples were sequentially saturated with water of three different $\mathrm{NaCl}$ concentrations. We performed complex electrical measurements on homogeneous and three-layered unconsolidated sands during drainage to investigate the behavior of the complex resistivity as a function of water saturation at the different salinities. We propose a new double-impedance relaxation model with parameters that decrease exponentially with increasing saturation. We investigate how well the model fits the measured complex electrical resistivity of the sand packs for all water saturation levels and the three salt concentrations and compare it with Archie's classic law. Note that Archie's law describes resistance at zero frequency, but we tested its behavior outside its range of validity to see if it still might be useful.

\section{EXPERIMENTS}

We performed electrical resistivity measurements on medium and fine unconsolidated quartz sands with a grain size distribution ranging from 350 to $420 \mu \mathrm{m}$ and from 150 to $175 \mu \mathrm{m}$, respectively. We considered one homogeneous sand pack and two packs with three layers. Table 1 lists the properties of the sand packs and the three-letter acronyms we will use for further reference. We filled the sample holder, having a diameter of $15 \mathrm{~cm}$ and a height of $9 \mathrm{~cm}$, with a dry sample and vibrated it for 15 minutes. The resulting porosity was $\phi_{m}=0.375 \pm 0.005$ for the medium-grained sand and $\phi_{f}=0.395 \pm 0.005$ for the fine-grained sand. A detailed description of the experimental setup and sample holder can be found in an earlier paper (Kavian et al., 2011).

To measure the electrical response of the sample, we put the sample holder inside a Faraday box. An ISCO pump is used to fill or drain the sample from the bottom with water. Initially the water has a $\mathrm{NaCl}$ concentration of $1 \mathrm{mmol} / \mathrm{L}$. For the next desaturation stage, we drained the sample with a constant flow rate of $2 \mathrm{~mL} / \mathrm{min}$ until air breakthrough prevented the pump from removing more water from the sample. The electrical resistivity measurements ran continuously during the drainage cycles. We repeated the whole

Table 1. Three-letter acronyms for the layered sand packs, with the grain size in microns for each of the three $3-\mathrm{cm}$ thick layers.

\begin{tabular}{cccc} 
Sample & Bottom layer & Middle layer & Top layer \\
\hline HCL & 350 & 350 & 350 \\
CFC & 350 & 150 & 350 \\
FCF & 150 & 350 & 150 \\
\hline
\end{tabular}

procedure for a $\mathrm{NaCl}$ solution concentration of $10 \mathrm{mmol} / \mathrm{L}$ and of $100 \mathrm{mmol} / \mathrm{L}$.

To measure the electrical impedance, we connected a precision component analyzer (Wayne-Kerr, 6640A) to the two end electrodes. It measured the amplitude $|Z|(\Omega)$ and phase angle $\psi(\mathrm{rad})$ of the complex impedance, $Z=|Z| e^{\mathrm{i} \psi}$, as a function of frequency. The complex impedance measurements provide the effective electrical resistivity, $\rho=\rho_{r}-\mathrm{i} \rho_{i}$, of the sample, given by $\rho=(A / d) Z$, where $d(\mathrm{~m})$ denotes the sample height and $A\left(\mathrm{~m}^{2}\right)$ denotes the sample cross-sectional area. We used air measurements on the empty holder (Kavian et al., 2011) to subtract the residual impedance, caused by the sample holder, background noise, and the connecting cables. In the following section, we propose a relaxation model with parameters that were found to follow an exponential relationship with saturation and fit it to the complex electrical resistivity measurements for varying saline-water saturations and various degrees of heterogeneity.

\section{MODEL}

Different authors have used different generalizations of the standard Cole-Cole model (Cole and Cole, 1941) to fit measured electrical spectra in the frequency domain (Davidson and Cole, 1951; Pelton et al., 1978; Vanhala, 1997; Dias, 2000; Kruschwitz et al., 2010; Weller et al., 2010). We present a model that can fit complex impedance measurements as a function of frequency and saturation. It combines a standard Cole-Cole model and a Debye term

$$
Z(\omega)=\frac{R_{1}}{1+\left(\mathrm{i} \omega \tau_{1}\right)^{\alpha_{1}}}+\frac{R_{2}}{1+\mathrm{i} \omega \tau_{2}} .
$$

The model is consistent with the coupled Cole-Cole model introduced by Kemna et al. (2000)

$$
Z(\omega)=R_{0}\left[1-\sum_{k=1}^{2} m_{k}\left(1-\frac{1}{1+\left(\mathrm{i} \omega \tau_{k}\right)^{\alpha_{k}}}\right)\right],
$$

where $R_{0}$ is the resistance at zero frequency, $m_{k}$ the chargeability, $\tau_{k}$ a relaxation time, and $\alpha_{k}$ a dispersion exponent. The latter describes the degree of frequency dependence for $Z$. When we take $m_{1}+m_{2}=1$ and $\alpha_{2}=1$ in equation 3 , we obtain equation 2 with alternative fitting parameters $R_{1}=m_{1} R_{0}$ and $R_{2}=m_{2} R_{0}$. The rationale behind this choice is minimization of the number of fitting parameters. The condition $m_{1}+m_{2}=1$ indicates that the impedance in the infinite-frequency limit becomes zero. The assumption of $\alpha_{2}=1$ reflects a classical parallel R-C circuit. Our choice reduces the number of fitting parameters from seven to five without loss of accuracy in the fit as demonstrated in our study of unconsolidated sand packs (Kavian et al., 2011). It was found that this five-parameter model superbly fits the complex electrical resistivity spectra measured under continuous flow conditions for given saturation and salinity.

The solution of the nonlinear weighted least-squares problem was accomplished by a Gauss-Newton method, using the pseudoinverse of the Hessian to accommodate possible singular behavior during the iterations. The inverse Hessian at convergence, multiplied by the weighted sum of squared errors and divided by the number of measurements minus the number of parameters, was taken as an estimate of the covariance matrix of the problem. 
The error estimates listed in the Table 2 correspond to the square-root of its diagonal values. This provides a rough estimate of the error in the parameters, ignoring correlations between them. When we cross-plotted the logarithm of four of the inverted parameters, $\log \left(R_{k}\right)$ and $\log \left(\tau_{k}\right)$ with $k=1,2$, as a function of saturation $\left(S_{w}\right)$, all trends were approximately linear. This suggests the following choice for the resistance $\left(R_{1}, R_{2}\right)$ and relaxation time $\left(\tau_{1}, \tau_{2}\right)$ :

$$
R_{k}=\exp \left(\mu_{k}+\beta_{k} S_{w}\right) \quad \text { and } \quad \tau_{k}=\exp \left(\gamma_{k}+\eta_{k} S_{w}\right),
$$

Table 2. Model parameters for the three unconsolidated sand samples (HCL, CFC, and FCF) obtained by fitting the model of equation 5 to the complex impedance data obtained during drainage for three different salt concentrations of 1 , 10, and $100 \mathrm{mmol} / \mathrm{L}$. RMSE represents the normalized root-mean-square error over the range of frequencies and water saturations, as defined in equation 6 . The numbers in parentheses are the estimated errors in the parameters.

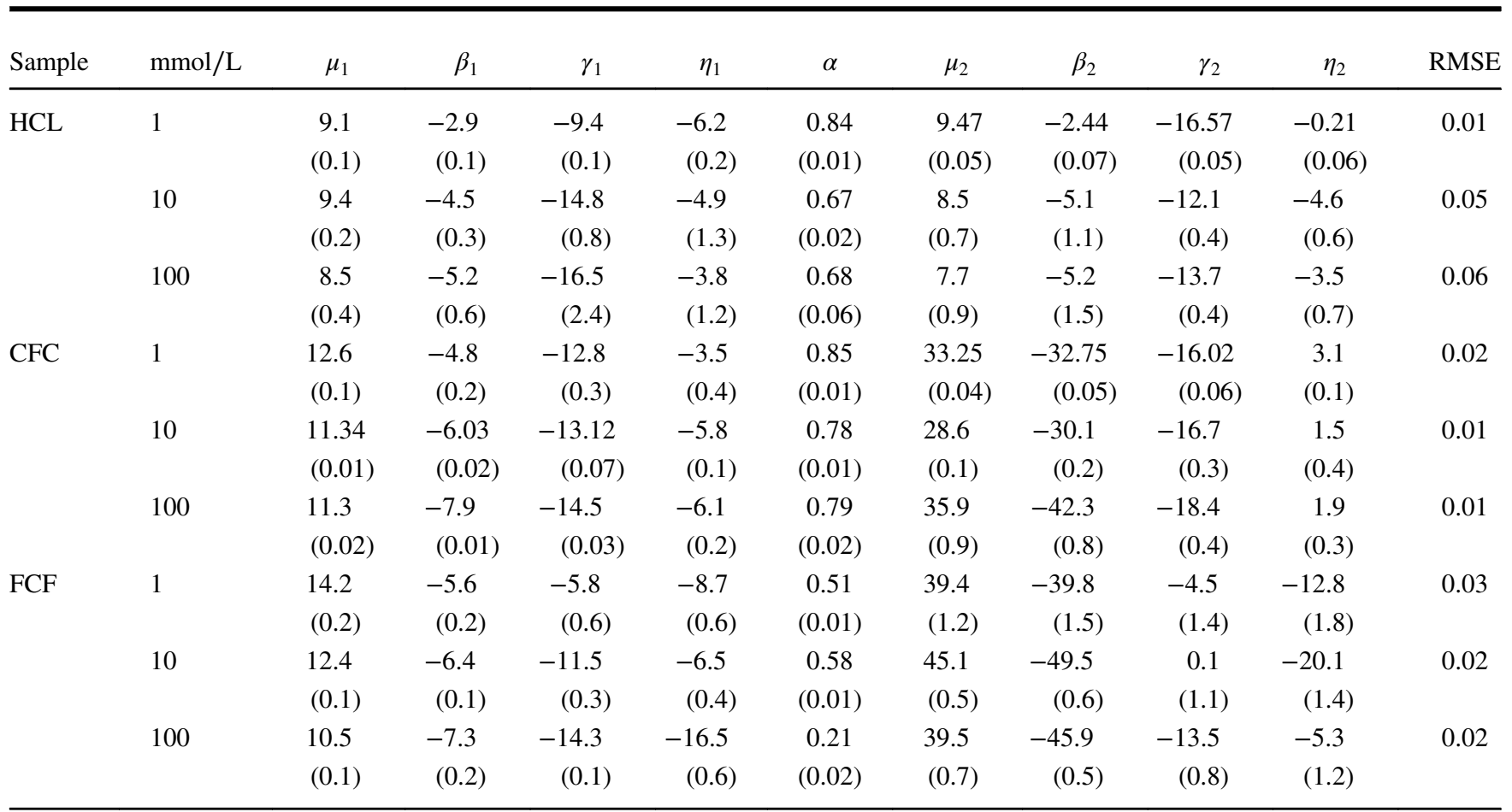

a)

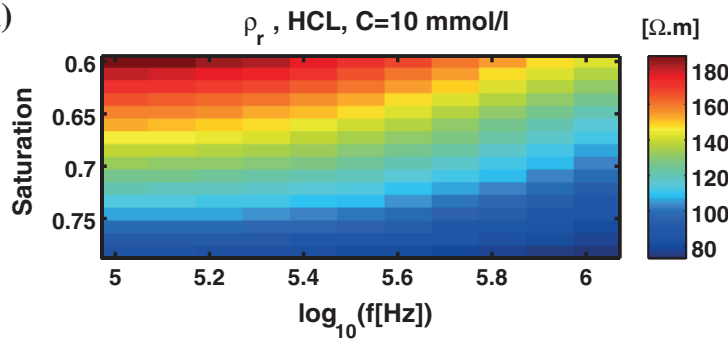

c)

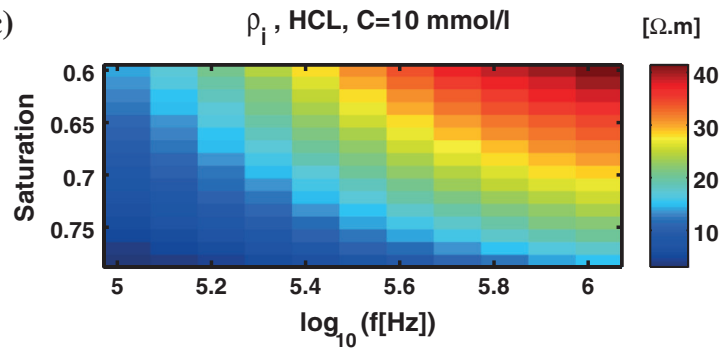

b)

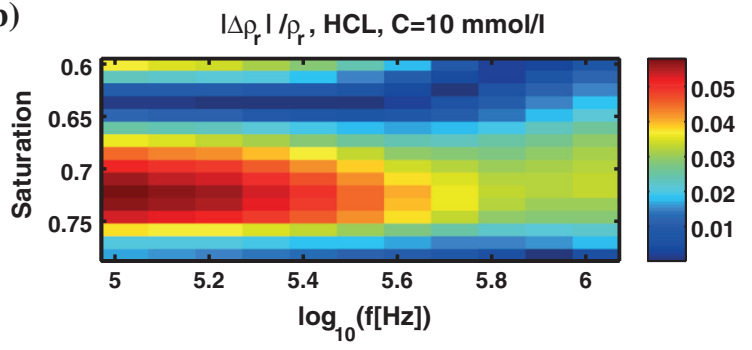

d)

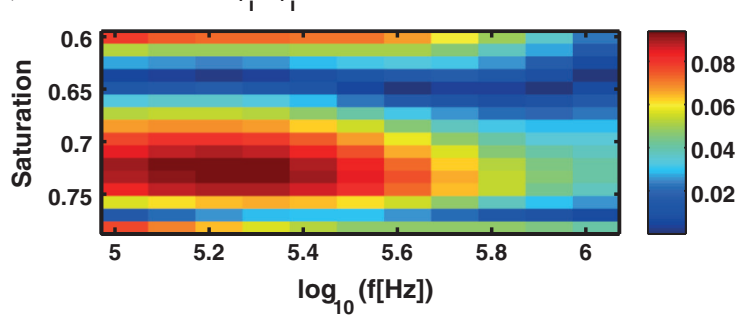

Figure 1. Images of the (a) real and (b) imaginary part of the electrical resistivity during drainage as a function of frequency and saturation for the homogeneous unconsolidated sand pack (HCL) with an average grain size of 350 micron. The $\mathrm{NaCl}$ concentration was $10 \mathrm{mmol} / \mathrm{L}$. On the right, we show the relative errors between the experimental data and our model for the (c) real and (d) imaginary part of the resistivity. 
a)

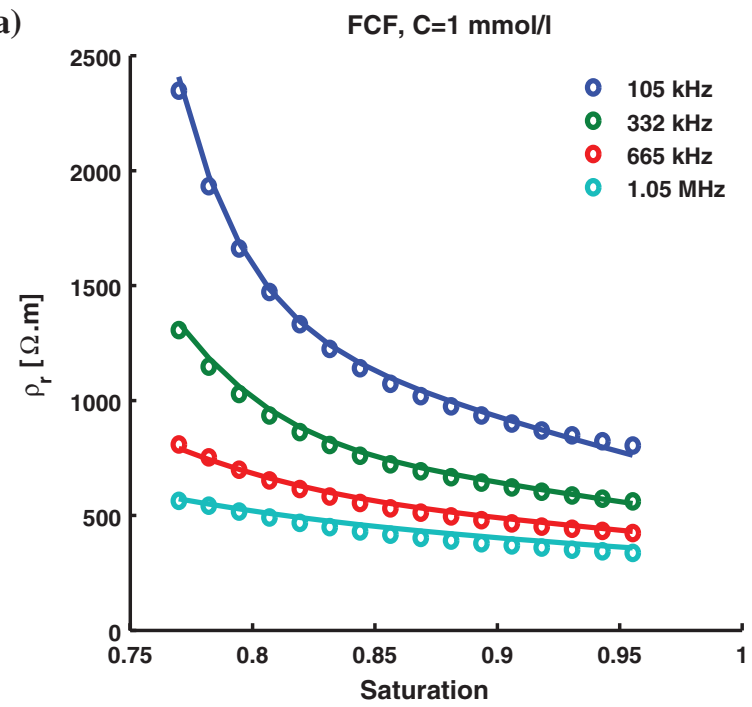

c)

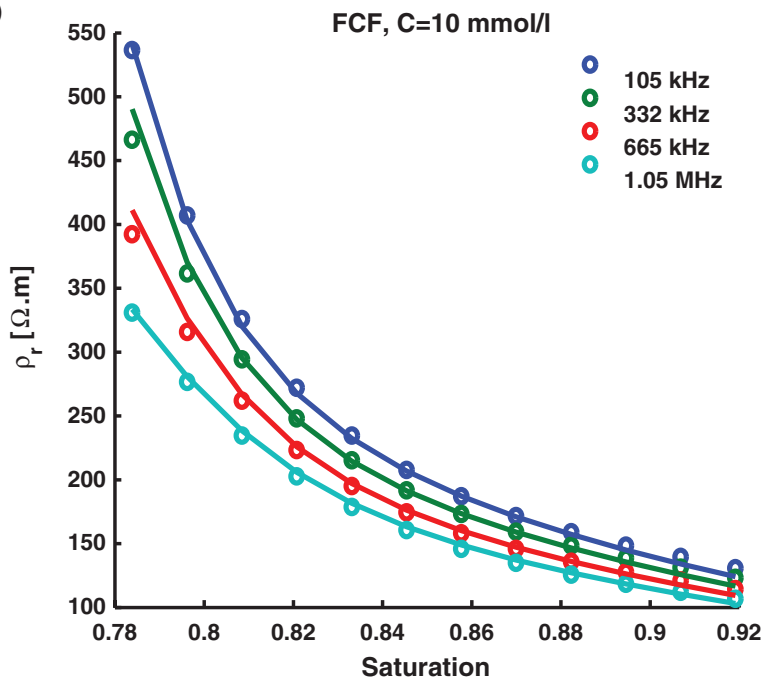

e)

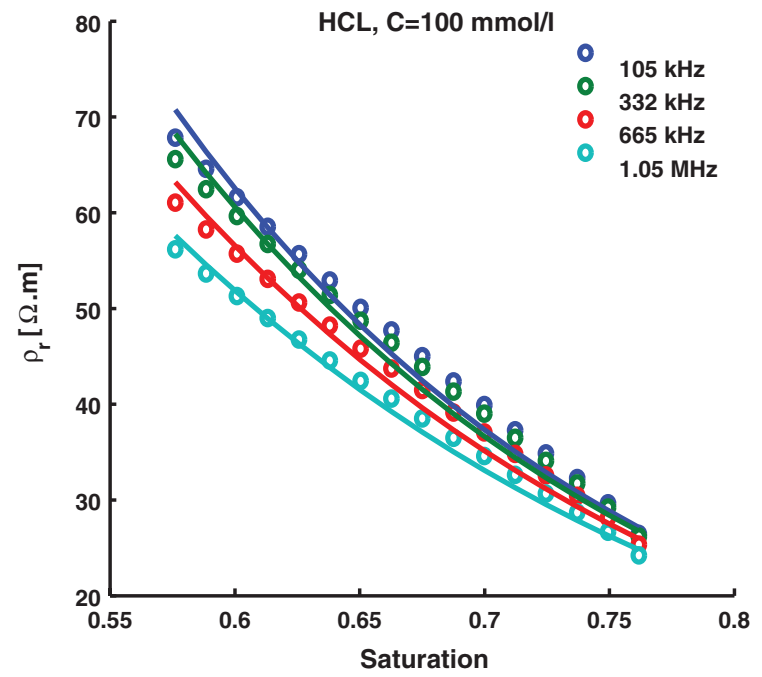

b)

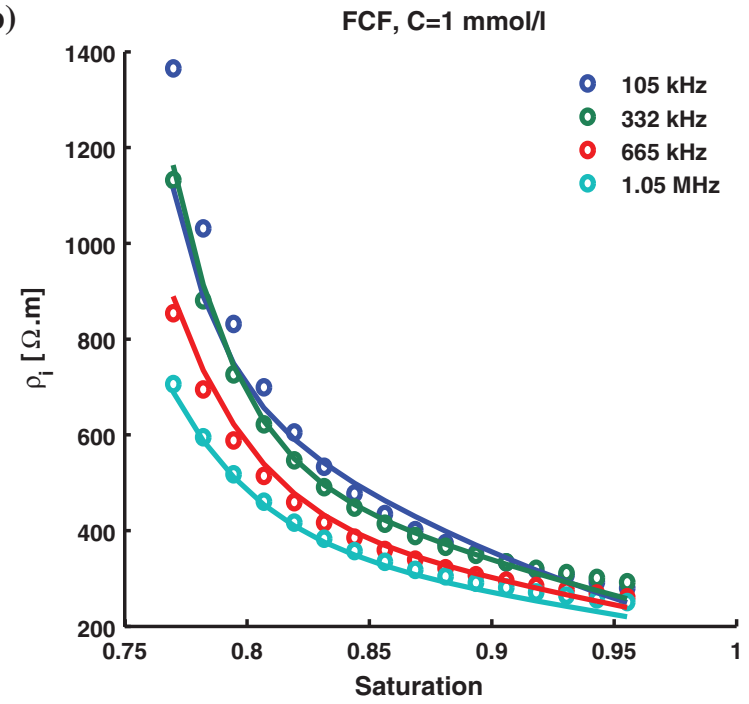

d)

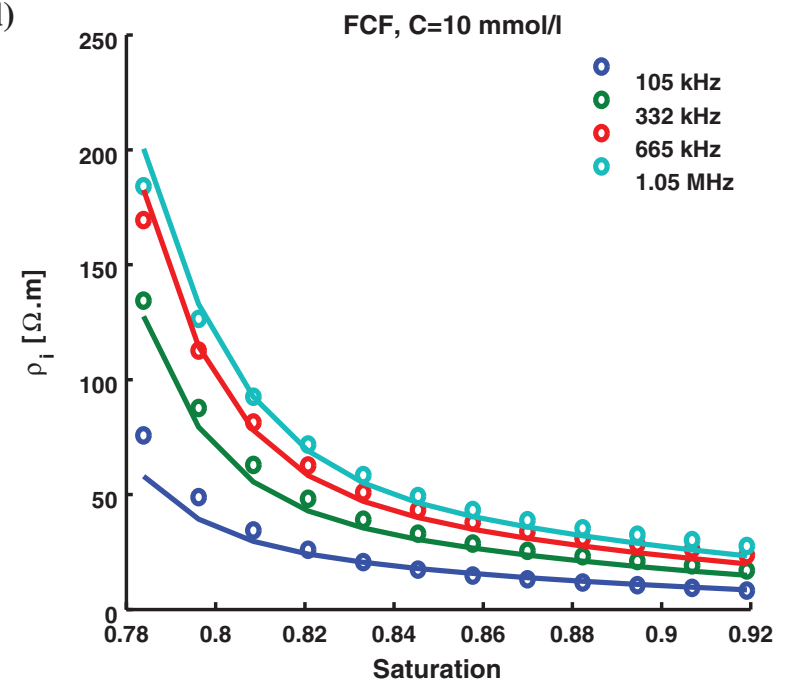

f)

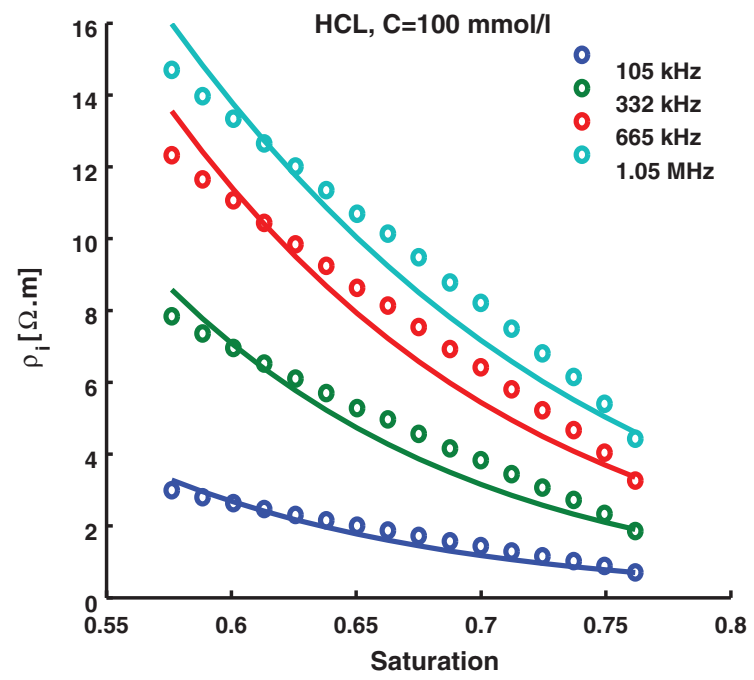

Figure 2. Variation of (a, c, and e) $\rho_{r}$ and (b, d, and f) $\rho_{i}$ with saturation for the FCF and HCL samples. The results are shown for NaCl concentrations of $(\mathrm{a}, \mathrm{b}) 1,(\mathrm{c}, \mathrm{d}) 10$, and $(\mathrm{e}, \mathrm{f}) 100 \mathrm{mmol} / \mathrm{L}$. The symbols denote the experimental results; the solid lines represent the data fits based on equation 5 . 
with new parameters $\mu_{k}, \beta_{k}, \gamma_{k}$, and $\eta_{k}$. Substitution into equation 2 yields the following model

$$
Z\left(\omega, S_{w}\right)=\frac{e^{\left(\mu_{1}+\beta_{1} S_{w}\right)}}{1+\left[\mathrm{i} \omega e^{\left(\gamma_{1}+\eta_{1} S_{w}\right)}\right]^{\alpha_{1}}}+\frac{e^{\left(\mu_{2}+\beta_{2} S_{w}\right)}}{1+\mathrm{i} \omega e^{\left(\gamma_{2}+\eta_{2} S_{w}\right)}} .
$$

For each $\mathrm{NaCl}$ concentration, we estimated the nine parameters by fitting the model to the experimental data as a function of frequency and water saturation. Note that we initially considered simple power-law models, but these produced much poorer fits than the exponential one. We performed joint inversion of the real and imaginary parts of the complex impedance measurements. Table 2 lists the estimated model parameters for the three unconsolidated sand packs, obtained from measurements during drainage, at the three $\mathrm{NaCl}$ concentrations of 1,10 , and $100 \mathrm{mmol} / \mathrm{L}$. To quantify

a)

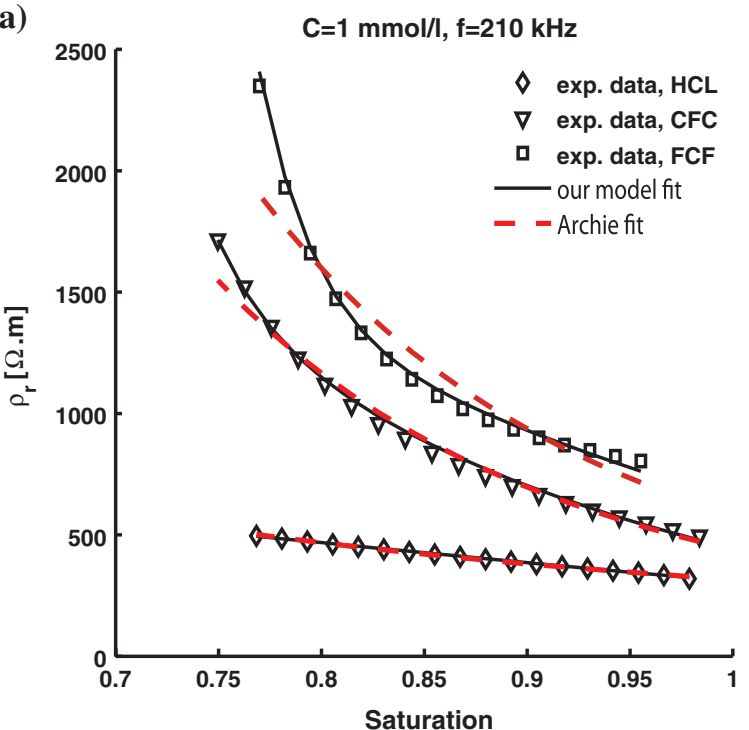

c)

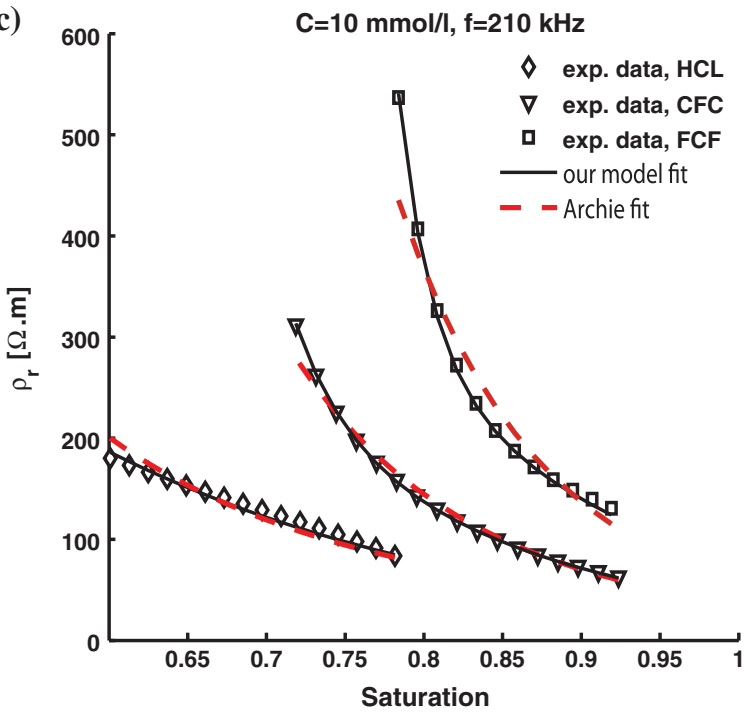

how well the model fits the complex impedance data over the full saturation and frequency ranges, we list the global root mean square error (RMSE),

$$
\operatorname{RMSE}=\left(\frac{\sum_{\omega, S_{w}}\left|\rho\left(\omega, S_{w}\right)-\rho^{\mathrm{obs}}\left(\omega, S_{w}\right)\right|^{2}}{\sum_{\omega, S_{w}}\left|\rho^{\mathrm{obs}}\left(\omega, S_{w}\right)\right|^{2}}\right)^{1 / 2} .
$$

The results have an error of less than $6 \%$ for all three $\mathrm{NaCl}$ solution concentrations.

Figure $1 \mathrm{a}$ and $1 \mathrm{~b}$ displays the real and imaginary parts of the resistivity data and the associated relative error (Figure 1c and 1d), given by

b)

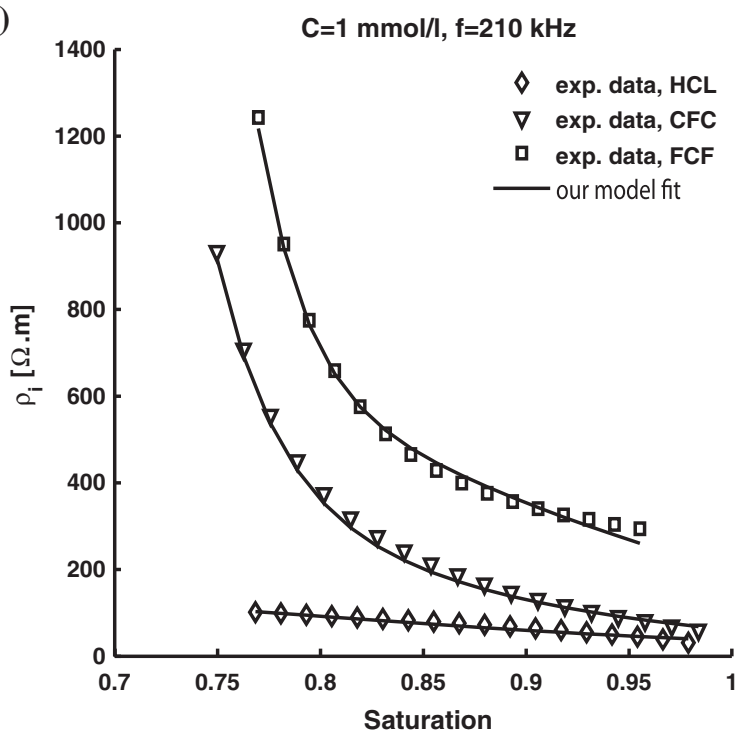

d)

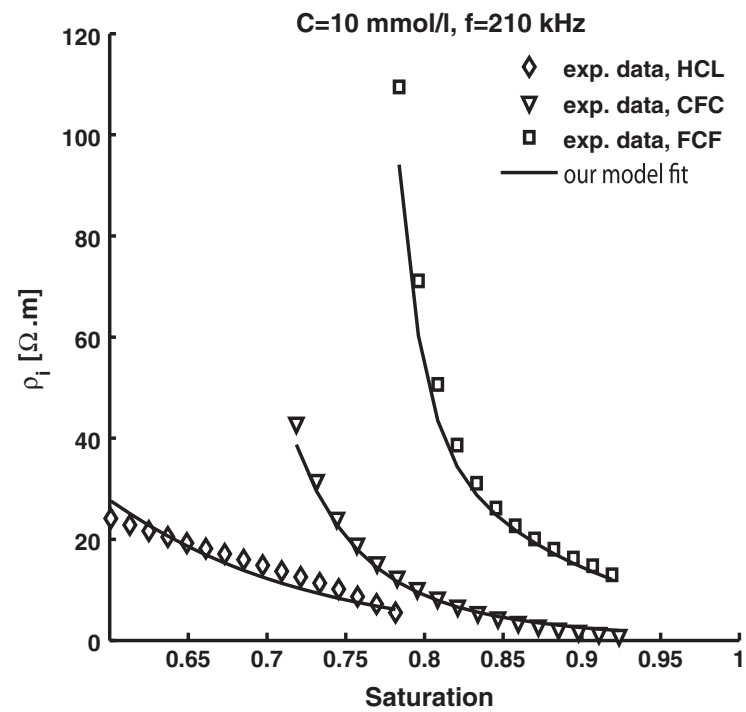

Figure 3. Variation of $(\mathrm{a}, \mathrm{c}) \rho_{r}$ and $(\mathrm{b}, \mathrm{d}) \rho_{i}$ with saturation for the HCL, CFC, and FCF samples. The results correspond to NaCl concentrations of $(\mathrm{a}, \mathrm{b}) 1$ and $(\mathrm{c}, \mathrm{d}) 10 \mathrm{nmol} / \mathrm{L}$. The symbols denote the experimental results, the solid lines represent the data fits. The red dashed lines show the fit of $\rho_{r}$ with Archie's model. 


$$
\frac{\left|\Delta \rho_{r, i}\right|}{\left|\rho_{r, i}\right|}=\frac{\left|\rho_{r, i}\left(\omega, S_{w}\right)-\rho_{r, i}^{\mathrm{obs}}\left(\omega, S_{w}\right)\right|}{\left|\rho_{r, i}^{\mathrm{obs}}\left(\omega, S_{w}\right)\right|},
$$

for the homogeneous medium-grained size sand sample (HCL). From the $10 \mathrm{mmol} / \mathrm{L}$ sand packs, this sample showed the largest overall data RMSE. The relative error in fits of the real and imaginary parts of the electrical resistivity is satisfactory at all saturation levels. For other layered samples, fits (not shown here) are much better as indicated by the fitting errors (RMSE) in Table 2.

\section{RESULTS}

\section{Frequency and saturation dependence}

Figure 2 represents the plots of the real and imaginary parts of the electrical resistivity versus water saturation for the three-layered (FCF) and homogeneous medium-grained (HCL) sand samples at frequencies of $105 \mathrm{kHz}, 332 \mathrm{kHz}, 665 \mathrm{kHz}$, and $1.05 \mathrm{MHz}$, in each plot for a single $\mathrm{NaCl}$ solution concentration. We use

$$
\rho_{r}=(A / d) \operatorname{Re}\left(Z\left(\omega, S_{w}\right)\right), \quad \rho_{i}=(A / d) \operatorname{Im}\left(Z\left(\omega, S_{w}\right)\right),
$$

to convert the modeled impedance values to real and imaginary resistivities and plot the results together with the experimental results to show the quality of fit. The solid lines in Figure 2 display the modeled results for the drainage process. This model excellently reproduces the saturation dependence observed in the real and imaginary parts of the electrical resistivity. Note that the graphs presented here illustrate the worst cases for the three-layered (FCF) and homogeneous (HCL) sand samples. The three-layered sample at $1 \mathrm{mmol} / \mathrm{L}$ salinity is significantly better described (Figure 2a and $2 \mathrm{~b}$ ) than the homogeneous sample at $100 \mathrm{mmol} / \mathrm{L}$ (Figure 2e and 2f). This suggests that heterogeneity is better captured by our model than high values of salinity. Figure $2 \mathrm{a}$ and $2 \mathrm{~b}$ shows the real and imaginary parts of the electrical resistivity versus saturation for the three-layered sand sample (FCF) at the lowest $\mathrm{NaCl}$ solution concentration. We found a RMSE of $3 \%$. The real part of the resistivity as a function of saturation decreases with increasing frequency, a phenomenon that is similar to that in the imaginary part, but only for this sample. For the other samples and all salt concentrations, the real part of the resistivity decreases with increasing frequency. An increase of the salinity by another order of magnitude leads to smaller values for the complex resistivity versus saturation, with a RMSE of $2 \%$, as shown in Figure $2 \mathrm{c}$ and $2 \mathrm{~d}$. The fit for the imaginary part at the lowest frequency is better than the fit for $1 \mathrm{mmol} / \mathrm{L}$ (compare Figure $2 \mathrm{~b}$ and $2 \mathrm{~d}$ ). At a $\mathrm{NaCl}$ concentration of $1 \mathrm{mmol} / \mathrm{L}$, the maximum value of $\rho_{r}$ at $105 \mathrm{kHz}$ is four times higher than the maximum value at $1.05 \mathrm{MHz}$ - see Figure 2a. We observe a factor of less than two at a $10 \mathrm{mmol} / \mathrm{L}$ salt concentration in Figure 2c.

\section{Archie's law and exponential model}

Figure 3 shows the real and imaginary parts of the electrical resistivity data versus water saturation at a frequency of $210 \mathrm{kHz}$ for $1 \mathrm{mmol} / \mathrm{L}$ and for $10 \mathrm{mmol} / \mathrm{L}$ together with our model fit (equation 5) and the fit with Archie's law (equation 1). Each plot shows desaturation results for the three different sand packs, homogeneous sand (HCL), and the three-layered sands CFC and
FCF. By comparing the results in Figure $3 \mathrm{a}$ and $3 \mathrm{c}$, two observations can be made. First, the lowest resistivity observed in all salinities for the homogeneous medium-grained sand could be due to the high permeability where Archie's law and our exponential model agree very well to the data at the lowest salinity. At the next $\mathrm{NaCl}$ concentration of $10 \mathrm{mmol} / \mathrm{L}$, we observe a poor fit of Archie's model in comparison to our exponential model, shown for the HCL sample in Figure 3c. When the fine-grain fraction increases, as in the FCF sample, Archie's model cannot estimate the variations in electrical resistivity versus saturation. This could point to the possibility of grain surface counterion migration as a cause for bulk resistivity at these low ion concentrations (Revil and Glover, 1998), which Archie's law cannot capture but our exponential relaxation model can.

\section{CONCLUSIONS}

The most commonly used formula for describing the variation in electrical resistivity with saturation, introduced by Archie (1942), has the electrical resistivity proportional to $S_{w}^{-n}$, where $n$ is referred to as the saturation exponent. Our results show that the variation in electrical resistivity with $S_{w}$ and salinity is more complicated. The complexity increases when we take the frequency dependence of the electrical resistivity into account. The new complex resistivity model captures these complexities very well. Although Archie's law describes resistance at zero frequency, it still is able to fit the real part of our measured complex resistivity at higher frequencies for a homogeneous sample, but less well for a heterogeneous sample. Our proposed model captures the full complex resistivity response as a function of frequency and water saturation. We have shown that it can be applied to heterogeneous samples over a range of saturations and salt concentrations. It remains to be investigated if the model will be able to describe samples with arbitrary grain size distributions.

\section{ACKNOWLEDGMENTS}

The authors thank Delft Earth and Shell for funding the project.

\section{REFERENCES}

Archie, G. E., 1942, The electrical resistivity log as an aid in determining some reservoir characteristics: Transactions of the American Institute of Mining, Metallurgical, and Petroleum Engineers, 146, 54-62.

Binley, A., L. D. Slater, M. Fukes, and G. Cassiani, 2005, Relationship between spectral induced polarization and hydraulic properties of saturated and unsaturated sandstone: Water Resources Research, 41, 1-13, doi: 10 1029/2005WR004202.

Cassiani, G., A. Kemna, A. Villa, and E. Zimmermann, 2009, Spectral induced polarization for the characterization of free-phase hydrocarbon contamination of sediments with low clay content: Near Surface Geophysics, 7, 547-562, doi: 10.3997/1873-0604.2009028.

Cole, K. S., and R. H. Cole, 1941, Dispersion and absorption in dielectrics: Journal of Chemical Physics, 9, 341-351, doi: 10.1063/1.1750906.

Davidson, D. W., and R. H. Cole, 1951, Dielectric relaxation in glycerol, propylene glycol and n-propanol: Journal of Chemical Physics, 19, 1484-1490, doi: $10.1063 / 1.1748105$.

Dias, C. A., 2000, Developments in a model to describe low-frequency electrical polarization of rocks: Geophysics, $\mathbf{6 5}, 437-451$, doi: 10.1190/1 .1444738 .

Ghorbani, A., P. C. Cosenza, A. Revil, M. Zamora, M. Schmutz, N. Florsch, and D. Jougnot, 2009, Non-invasive monitoring of water content and textural changes in clay-rocks using spectral induced polarization: A laboratory investigation: Applied Clay Science, 43, 493-502, doi: 10.1016/j.clay.2008.12.007.

Jougnot, D., A. Ghorbani, A. Revil, P. Leroy, and P. Cosenza, 2010, Spectral induced polarization of partially saturated clay-rocks: A mechanistic approach: Geophysical Journal International, 180, 210-224, doi: 10 $.1111 / \mathrm{gji}$.2010.180.issue-1. 
Kavian, M. E. C. Slob, and W. A. Mulder, 2011, Hysteresis in the nonmonotonic electric response of homogeneous and layered unconsolidated sands under continuous flow conditions with water of various salinities, $100 \mathrm{kHz}$ to $2 \mathrm{MHz}$ : Journal of Geophysical Research, 116, B08214, doi: 10.1029/2010JB008083.

Kemna, A., A. Binley, A. Ramirez, and W. Daily, 2000, Complex resistivity tomography for enviromental applications: Chemical Engineering Journal, 77, 11-18, doi: 10.1016/S1385-8947(99)00135-7.

Kruschwitz, S., A. Binley, D. Lesmes, and A. Elshenawy, 2010, Textural controls on low-frequency electrical spectra of porous media: Geophysics, 75, no. 4, WA113-WA123, doi: 10.1190/1.3479835.

Park, S. K., and S. K. Dickey, 1989, Accurate estimation of conductivity of water from geoelectrical measurements, a new way to correct for clay: Ground Water, 27, 786-792, doi: 10.1111/gwat.1989 .27.issue-6.

Pelton, W. H., S. H. Ward, P. G. Hallof, W. R. Sill, and P. H. Nelson, 1978, Mineral discrimination and removal of inductive coupling with multifrequency IP: Geophysics, 43, 588-609, doi: 10.1190/1.1440839.

Revil, A., and P. W. J. Glover, 1998, Nature of surface electrical conductivity in natural sands, sandstones, and clays: Geophysical Research Letters, 25 , 691-694, doi: 10.1029/98GL00296.
Rink, M., and J. R. Schopper, 1974, Interface conductivity and its implication to electric logging: Transactions of the SPWLA 15th Annual Logging Symposium, 1-15.

Titov, K., A. Kemna, A. Tarasov, and H. Vereecken, 2004, Induced polarization of unsaturated sands determined through time-domain measurements: Vadose Zone Journal, 3, 1160-1168.

Ulrich, C., and L. D. Slater, 2004, Induced polarization measurements on unsaturated, unconsolidated sands: Geophysics, 69, 762-771, doi: $10.1190 / 1.1759462$.

Vanhala, H., 1997, Mapping oil-contaminated sand and till with the spectral induced polarization (SIP) method: Geophysical Prospecting, 45, 303-326, doi: 10.1046/j.1365-2478.1997.00338.x.

Vinegar, H. J., and M. H. Waxman, 1984, Induced polarization of shaly sands: Geophysics, 49, 1267-1287, doi: 10.1190/1.1441755.

Waxman, M. H., and L. J. M. Smits, 1968, Electrical conductivities in oil-bearing shaly sands: Society of Petroleum Engineers Journal, 8, 107-122, doi: 10.2118/1863-A.

Weller, A., L. Slater, S. Nordsiek, and D. Ntarlagiannis, 2010, On the estimation of specific surface per unit pore volume from induced polarization: A robust empirical relation fits multiple data sets: Geophysics, 75, no. 4, WA105-WA112, doi: 10.1190/1.3471577. 